\title{
Laparoscopic Inguinal Ligament Suspension for Vaginal Vault Prolapse: Video Step-by-Step
}

\author{
Chunbo Li, MD \\ Department of Gynaecology and Obstetrics, Shanghai First Maternity and Infant Hospital, \\ Tongji University School of Medicine, Shanghai, China.
}

\section{Zhiyuan Dai, MD}

Department of Gynaecology and Obstetrics, Shanghai First Maternity and Infant Hospital, Tongji University School of Medicine, Shanghai, China.

E-mail: daizhiyuan@51mch.com

Huimin Shu, MD

Department of Gynaecology and Obstetrics, Shanghai First Maternity and Infant Hospital, Tongji University School of Medicine, Shanghai, China.

\section{Kai Zhang, PhD}

Department of Anatomy, Tongji University School of Medicine, Shanghai China.

\section{Chenyun Dai, PhD}

University of North Carolina at Chapel Hill, Chapel Hill, North Carolina.

(C) Mary Ann Liebert, Inc. DOI: 10.1089/vor.2017.0434
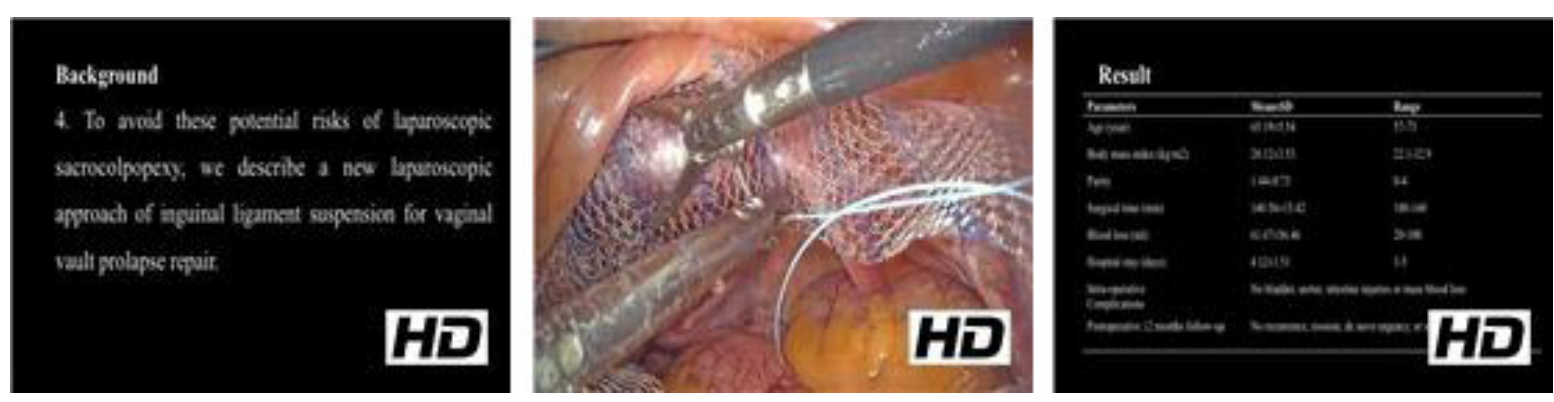

\section{Abstract}

Introduction: Posthysterectomy vaginal vault prolapse (PVVP), defined as prolapse recurrence after primary hysterectomy, represents a distressing issue for both patients and surgeons. ${ }^{1-3}$ Various surgical procedures have been described to correct PVVP. Until now, sacrocolpopexy performed abdominally, laparoscopically, or robotically is accepted as the gold standard procedure for PVVP with success rates of $74-98 \% .^{4,5}$ However, the approach is often accompanied by intraoperative complications (massive blood loss and ureter/intestine injuries) and postoperative complications (mesh erosion, defecation dysfunction, postoperative ileus, ureteral obstruction, and dyspareunia). ${ }^{5}$ In addition, dissection at the level of the promontory may be challenging, particularly in obesity, severe abdominal adhesion, sigmoid megacolon, low position of the left common iliac vein, or large anatomical variation existing. These may be associated with serious neurological or ureteral morbidity as well as life-threatening vascular injury. ${ }^{6}$ In this video, we introduced a surgical technique of laparoscopic inguinal ligament suspension (LILS). The objective of the video is to present the detailed steps of LILS to achieve effective repair for PVVP.

Materials and Methods: After a vaginal vault cone was inserted into the vagina, the anastomotic stoma of vaginal vault was exposed. Then, surface membrane of the vaginal vault was opened and both bladder and rectum were mobilized away from the vagina down to the level of the trigone and the levator ani plate, creating the vesicovagina space anteriorly and the rectovaginal space posteriorly, 
respectively. After that, the bottom of a cross-shaped polypropylene mesh was sutured to the top of the vaginal fornix and two short arms of the mesh were sutured to the anterior and posterior vaginal wall, respectively. Then, the suspension area of inguinal ligament between the inlet of inguinal canal and anterior superior iliac spine was identified. The portion of inguinal ligament that was $1-2 \mathrm{~cm}$ distance from anterior superior iliac was exposed completely. An extraperitoneal tunnel between the suspension points and the vaginal vault was created. The long arms of the mesh were introduced along the round ligament to the suspension points and fixed into inguinal ligament/fascia. At last, the peritoneal incision was closed.

Results: The LILS was effectively completed in 10 patients. The mean age was 65.2 years (range $57.0-73.0$ years), the mean body mass index was $28.1 \mathrm{~kg} / \mathrm{m}^{2}$ (range $22.1-32.9$ ) and the mean parity was 1.4 (range 0-4). The mean surgical time was $140.6 \pm 15.4$ minutes, the mean blood loss was $61.7 \pm 56.5 \mathrm{~mL}$, and the mean hospital stay was 4.2 days (range $3-5$ days). No intraoperative complications occurred. After a 12-month follow-up, both the anatomical correction and subjective symptoms such as symptom severity, quality of life, and sexual activity presented significant improvement. There was no recurrence, erosion, de novo urgency, or urinary incontinence at 12-month follow-up.

Conclusions: Our preliminary outcome indicated that LILS was a safe and efficient option for the treatment of PVVP, and might be an alternative treatment for patients with vaginal vault prolapse, especially for patients presenting potential difficulties or risks to access to promontory. Further studies are warranted to determine the role of LILS for prolapse repair.

Acknowledgment: The Science and Technology Commission of Shanghai Municipality (17411967900); the Health and Family Planning Committee of PuDong New Area of Shanghai (PW2015D-9).

No competing financial interests exist.

Runtime of video: 6 mins 21 secs

Keywords: pelvic organ prolapse, laparoscopic technique, inguinal ligament, laparoscopic inguinal ligament suspension

\section{Cite this video}

Chunbo Li, Zhiyuan Dai, Huimin Shu, Kai Zhang, Chenyun Dai, Laparoscopic Inguinal Ligament Suspension for Vaginal Vault Prolapse: Video Step-by-Step, Videoscopy. 2017, DOI: 10.1089/ vor.2017.0434.

\section{References}

1. Ismail S, Duckett J, Rizk D, et al. Recurrent pelvic organ prolaspe: Internation Urogynecological Association Research and Development Committee opinion. Int Urogynecol J 2016;27:1619-1632.

2. Dällenbach P, Kaelin-Gambirasio I, Jacob S, Dubuisson JB, Boulvain M. Incidence rate and risk factors for vaginal vault prolapse repair after hysterectomy. Int Urogynecol J 2008;19:1623-1629.

3. Ganatra AM, Rozet F, Sanchez-Salas R, Barret E, Galiano M, Cathelineau X, Vallancien G. The current status of laparoscopic sacrocolpopexy: A review. Eur Urol 2009;55:1089-1103.

4. Parkes IL, Shveiky D. Sacrocolpopexy for treatment of vaginal apical prolspe: Evidence-based surgery. J Minim Invasive Gynecol 2014;21:546-557.

5. Germain A, Thibault F, Galifet M, Scherrer ML, Ayav A, Hubert J, Brunaud L, Bresier L. Long-term outcomes after totally robotic sacrocolpopexy for treatment of pelvic organ prolapse. Surg Endosc 2013;27:525-539.

6. Anand M, Weaver AL, Fruth KM, Gebhart JB. Factors influencing selection of vaginal, open abdominal, or robotic surgery to treat apical vaginal vault prolapse. Female Pelvic Med Reconstr Surg 2016;22:236-242. 